ISSN electrónico: 2602-8069

\title{
PROPUESTA TEÓRICA Y METODOLÓGICA DEL PROCESO DE INDUCCIÓN LABORAL DE PERSONAL DOCENTE EN INSTITUCIONES DE EDUCACIÓN SUPERIOR
}

Theoretical and methodological proposal of the process of labor induction of teaching staff in institutions of higher education

María Belén Rueda Reyes ${ }^{1}$

Universidad Tecnológica Equinoccial del Ecuador.

belen.rueda@ute.edu.ec

Fecha de recepción del artículo: 21/07/2017

Fecha de aceptación definitiva: 07/05/2018

\footnotetext{
${ }^{1}$ Magíster en Gestión del Talento Humano. Docente de la Facultad de Arquitectura Artes y Diseño de la Universidad Tecnológica Equinoccial del Ecuador. 


\title{
María Belén Rueda Reyes \\ Propuesta teórica y metodológica del proceso de inducción laboral de personal docente en instituciones de educación superior
}

\section{RESUMEN}

El presente artículo tiene por objetivo proponer un proceso base de inducción laboral dirigido a personal docente de instituciones de educación superior. La propuesta busca convertirse en una herramienta flexible que pueda ser adaptada a las necesidades particulares de la institución y de los propios docentes, con lo que se aportará a la construcción de mejores ambientes de trabajo, disminución de las tasas de rotación de personal, mayor compromiso y fidelidad, así como el cumplimiento de los objetivos estratégicos institucionales, lo que beneficia tanto a las organizaciones como a los docentes, la comunidad universitaria y, en última instancia, a la sociedad. El ensayo, cualitativo y descriptivo, con base en una revisión bibliográfica, realiza una aproximación teórica al proceso de inducción, argumenta su tratamiento como función de la administración de talento humano, define su impacto en las organizaciones y, posteriormente, desarrolla una revisión analítica de las particularidades de la práctica docente universitaria. Las consideraciones previas sirven como insumo para realizar una propuesta de un modelo de inducción y orientación que puede aplicarse en universidades, escuelas politécnicas y demás instituciones de educación superior, buscando ser suficientemente adaptable a las particularidades de instituciones, facultades y personas.

Palabras clave: Inducción, Docente universitario, Administración de talento humano, Instituciones de educación superior, Proceso de recursos humanos.

\begin{abstract}
The main objective of this article is to propose a basic labor induction process aimed at teaching staff of higher education institutions. The proposal seeks to become a flexible tool that can be adapted to the particular needs of institutions and the teachers themselves, which will contribute to the construction of better work environments, decrease in staff turnover rates, greater commitment and fidelity, as well as the fulfillment of institutional strategic objectives, which benefits organizations, teachers, the university community and, ultimately, the whole society. The essay, qualitative and descriptive, based on a bibliographical study, makes a theoretical approach to the induction process, argues its treatment as a function of the administration of human talent, defines its impact on organizations and, subsequently, develops an analytical review of the particularities of university teaching practice. The previous considerations serve as input to make a proposal of an induction and orientation model that can be applied in universities, polytechnic schools and other institutions of higher education, seeking to be sufficiently able to be adapted to the particularities of institutions, faculties and people.
\end{abstract}

Keywords: Induction, University teachers, Talent management, Institutions of higher education, Process of human resources.

\section{INTRODUCCIÓN}

Dentro de las actividades inherentes a la administración del talento humano se encuentran la inducción y la orientación inicial, como primera etapa para la adecuada colocación de los trabajadores en las diversas actividades que desarrollan las organizaciones (Chiavenato, 2009). La inducción tiene varios fines entre los que destaca la presentación de la cultura organizacional al nuevo trabajador y la adaptación a la institución, al trabajo con los compañeros y a las actividades 


\section{María Belén Rueda Reyes \\ Propuesta teórica y metodológica del proceso de inducción laboral de personal docente en instituciones de educación superior}

particulares a realizar (Grados, 2013). Esta adaptación es de suma importancia tanto para la institución, ya que aporta a la consolidación de la cultura organizacional y al cumplimiento de las normas, como para el propio trabajador recién integrado, por medio de mayor satisfacción con el puesto de trabajo y mejor conocimiento de lo que se espera de su colaboración. Por lo mencionado, lo que ha sido revisado extensamente por diversos autores, se considera que la inducción tiene un efecto positivo directo en el desempeño de los trabajadores.

Pese a lo descrito, en un estudio conducido por la autora de este documento, en 2015, en la Universidad Tecnológica Equinoccial de Ecuador, entre un grupo de 145 docentes de la Facultad de Arquitectura Artes y Diseño, se encontró que no existía una relación de causa-efecto entre la inducción recibida por los profesores universitarios y sus evaluaciones de desempeño en el puesto de trabajo (Rueda, 2015). Los resultados de ese estudio, sin duda, no pueden generalizarse y, como se busca describir en el presente artículo, podrían responder principalmente a las particularidades del puesto del docente universitario, así como a una disparidad en las actividades de inducción dentro de la institución -quizás, dentro de todas las instituciones- donde se llevó a cabo el estudio de campo.

Desde la antigüedad, la actividad docente ha mantenido una permanente heterogeneidad en cuanto a métodos y contenidos (Marrou, 2004), la misma que se ha visto multiplicada en la actualidad debido al desarrollo del conocimiento, la diferenciación entre teoría y práctica en los contenidos, la dinámica social interna, la cultura (tanto del contexto social como institucional) y la propia personalidad del docente (Borgobello, Peralta y Roselli, 2010). Esta multiplicidad de aristas que delimitan el trabajo del docente universitario se traduce, a su vez, en una diversidad de necesidades a ser consideradas en el desarrollo de un proceso efectivo y eficiente de inducción en las instituciones educativas superiores.

En virtud de lo señalado, en el presente artículo se realiza una aproximación teórica al proceso de inducción, de manera general, para revisar posteriormente las características del trabajo docente en las instituciones de educación superior, con el fin de establecer una serie de criterios base de la formulación de un proceso para la inducción de docentes, con el fin de aportar a la realización adecuada de las actividades de orientación inicial que favorezcan el desarrollo profesional de los maestros, así como el cumplimiento de los objetivos estratégicos de las instituciones universitarias. Tras el diseño de la propuesta, se presentan las conclusiones generales del artículo y se procede a la discusión de dichas conclusiones por medio de una revisión de literatura relacionada al tema y la comparación de los resultados.

\section{DESARROLLO DEL TEMA}

\section{Aproximación teórica al proceso de inducción}

En la actualidad, cada vez con mayor énfasis, las organizaciones (tanto públicas como privadas, en todas las ramas) buscan el desarrollo del talento humano como base de su éxito y apuestan por mudar la concepción clásica del trabajador como un recurso a la de ser considerado como asociado, de cuya formación y desarrollo depende la generación de un factor estratégico para el desarrollo de la productividad y la competitividad sostenibles (Mejía, Jaramillo y Bravo, 2006). De esta manera, las organizaciones buscan el mejoramiento de los procesos tocantes al talento humano para mejorar la selección del personal idóneo, la capacitación y el entrenamiento, la reducción de los índices de rotación de personal, entre otros objetivos de los que se encarga la denominada Gestión del Talento Humano. A esta rama de la administración también le compete el elemento central del presente artículo: la inducción. 


\section{María Belén Rueda Reyes \\ Propuesta teórica y metodológica del proceso de inducción laboral de personal docente en instituciones de educación superior}

Según Chiavenato (2009), se entiende por inducción u orientación ${ }^{22}$ al conjunto de actividades de entrenamiento inicial que se realiza, de manera formal e intensiva, con los nuevos miembros que han sido reclutados y seleccionados por la organización. Con la definición dada, coincide la de Mondy (2010) señala que la inducción constituye el esfuerzo inicial de capacitación y desarrollo que se dirige a los nuevos empleados para informarles acerca de la compañía, el puesto y el grupo de trabajo.

La base de la inducción radica en la necesidad de familiarizar a los trabajadores recién incorporados a la cultura de la empresa, así como adaptarlos a las estructuras organizacionales (autoridades, funcionarios, áreas y departamentos), además de brindar datos sobre el puesto de trabajo, los principales productos y servicios que ofrece la entidad, su misión, visión y objetivos, entre otros. En consecuencia, Luis Puchol (2007) señala que la inducción fundamentalmente tiene dos funciones: integrar e informar.

En lo que se refiere a la función integradora de la inducción del personal, constituye un método de aculturación de los nuevos participantes, de tal manera que comprendan, acepten y practiquen las rutinas de la organización (Chiavenato, 2007). La inducción, entonces, busca que el nuevo participante llegue a adaptarse a los componentes culturales como los comportamientos regulares de los individuos, las normas expresas y tácitas que se siguen en los grupos de trabajo, los valores dominantes de la organización, la filosofía de trabajo y el clima laboral (Rodríguez, 2006). De manera general, la inducción se convierte en un puente hacia la integración del nuevo trabajador a la organización y su cultura.

Por otra parte, la inducción del personal permite informar al trabajador de reciente ingreso acerca de las funciones que va a cumplir y lo que se espera de su colaboración, las relaciones de la estructura organizacional, la compensación y las prestaciones, las normas de seguridad, la distribución física de las instalaciones, y demás datos que servirán al trabajador para realizar sus actividades (Puchol, 2007). En este sentido, la inducción constituye la primera acción de capacitación de los nuevos trabajadores.

La inducción tiene una importancia sustancial para generar lazos institucionales sólidos y presenta beneficios tanto para las organizaciones como para los empleados. Para la organización, como comenta Alles (2006), la inducción representa un esfuerzo consciente y una inversión para acelerar la plena integración del colaborador en el puesto de trabajo, además de dar seguimiento a dicha integración; esto se traduce en beneficios como una menor rotación de personal, mejoramiento de la curva de aprendizaje, realización eficaz y eficiente de las actividades y, en general, lograr mayores niveles de productividad y competitividad (Mejía, Jaramillo y Bravo, 2006). Por otro lado, para los colaboradores, un óptimo proceso de inducción los beneficia en lo relativo a su satisfacción dentro de las organizaciones, la comodidad en el puesto de trabajo, la reducción de los riesgos inherentes a sus actividades y el desempeño de sus funciones (Puchol, 2007).

Ahora bien, se considera oportuno argumentar dos afirmaciones que, hasta el momento, se han dado por sentadas: 1) que la inducción es responsabilidad del área de talento humano (también denominada de recursos humanos); y, 2) que la inducción constituye un proceso.

Con respecto a la primera afirmación, se parte de la premisa de que la inducción se relaciona directamente con otras funciones que corresponden al área de talento humano como son el reclutamiento y la selección, la organización del personal, el desarrollo de los trabajadores y los procesos para retener a las personas. La inducción

\footnotetext{
${ }^{2}$ Pese a las múltiples denominaciones como adscripción, incorporación, acogida u orientación (Puchol, 2007), se ha optado por usar, a lo largo del artículo, el término "inducción" debido a que es comúnmente utilizado en el contexto ecuatoriano. 


\section{María Belén Rueda Reyes \\ Propuesta teórica y metodológica del proceso de inducción laboral de personal docente en instituciones de educación superior}

se ubicaría en una posición central debido a que, en primer lugar, es consecuencia de los procesos para integrar personas que involucran las acciones de reclutamiento y selección; por otra parte, a lo largo de la inducción se informa a la nueva incorporación acerca de los procesos para organizar a las personas, incluyendo el diseño del puesto y las condiciones de evaluación de desempeño, así como sobre los procesos para recompensar al personal entre las que están las remuneraciones, prestaciones e incentivos. Por último, la inducción forma parte o está relacionada con otros conjuntos de procesos para desarrollar (como la capacitación y formación), retener (seguridad y salud en el trabajo) y auditar (como los sistemas de evaluación) a las personas (Chiavenato, 2009). La relación descrita se presenta en el modelo de los seis procesos de gestión de talento humano propuesto por el mencionado autor que se ilustra en la Figura 1:

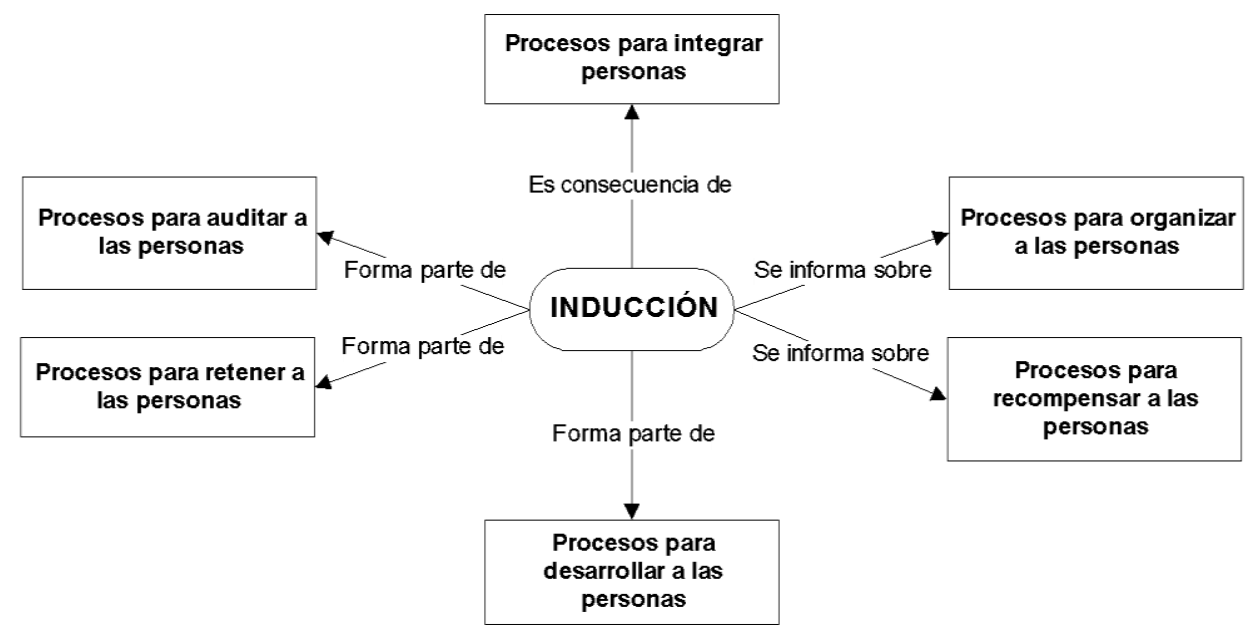

Figura 1. Inducción dentro de la gestión de talento humano. Esta figura representa la relación de la inducción con el modelo de los seis procesos que conforman las actividades de la función de talento humano, según propone Chiavenato (2009)

La relación directa con las actividades y procesos correspondientes a la gestión de talento humano hace que la inducción también corresponda a esta área en el conjunto de sus responsabilidades. No obstante lo anterior, cabe indicar que en muchos casos las acciones de integración y de información son desarrolladas por personal ajeno a esta área. Por ejemplo, Mondy (2010) señala que, si bien la inducción y sus actividades son supervisadas por recursos humanos, pueden ser adecuadamente desarrolladas por compañeros del nuevo trabajador en sus respectivas áreas debido a que suelen ser accesibles al personal de nuevo ingreso, tienden a mostrar un alto grado de simpatía, y tienen la experiencia organizacional y los conocimientos que los empleados nuevos necesitan. En suma, se observa que la inducción es responsabilidad del área de talento humano (esfera estratégica y administrativa de la inducción) pero puede ser desarrollada total o parcialmente por personal de otras áreas, de preferencia de la misma a la que ingresa el nuevo trabajador (esfera operativa de la inducción).

En lo referente a la segunda afirmación, esto es que la inducción se debe considerar como un proceso, se justifica en que coincide con la definición propia del término. Un proceso es, según lo describe Pérez (2010), una secuencia ordenada de actividades que permiten la transformación de elementos de entrada (inputs) en resultados (outputs). La inducción coincide con la descripción debido a que está constituida por una serie de acciones mutuamente relacionadas (bienvenida, información básica, presentación del área de trabajo, etc.) que transforman a un trabajador recientemente seleccionado en uno adaptado al puesto y dando inicio a su 


\section{Propuesta teórica y metodológica del proceso de inducción laboral de personal docente en instituciones de educación superior}

labor (Bozu, 2010), como se muestra en la Figura 2 que presenta un ejemplo de caracterización del proceso de inducción:

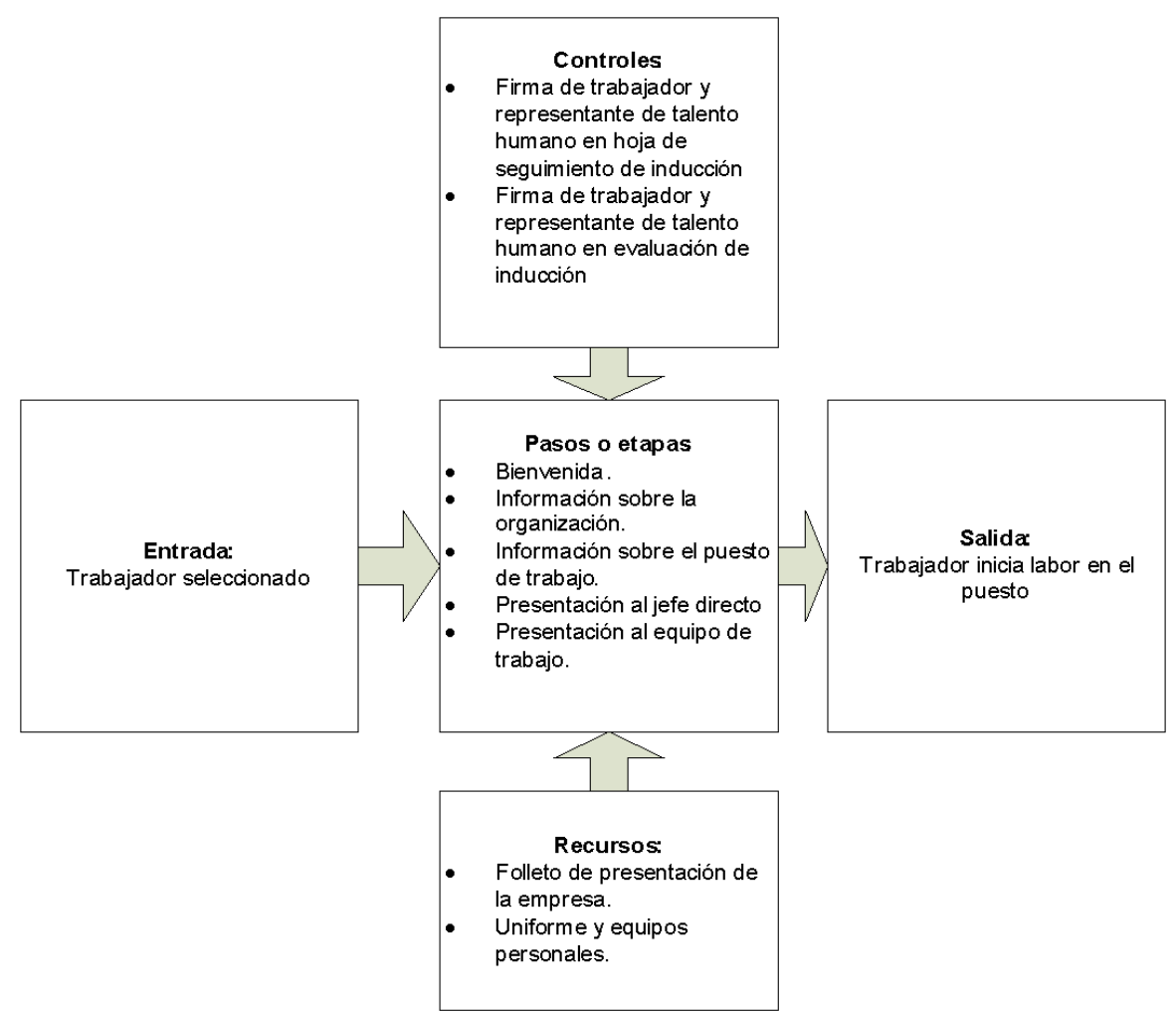

Figura 2. Caracterización del proceso de inducción de personal. Los elementos han sido adaptados de Bozu (2010) e ITT (2018).

Usualmente, la incorporación lleva consigo una serie de trámites administrativos y legales relacionados con la contratación; y, paralelamente, se añaden las acciones relacionadas con la acogida e incorporación del nuevo personal, lo que constituye el proceso de inducción que variará de acuerdo a la empresa, el puesto de trabajo, entre otros factores (Mondy, 2010). En el siguiente apartado se revisan ciertas particularidades relacionadas con el puesto de docente universitario.

\section{Características del trabajo como docente universitario}

En esta sección del artículo, se tratará de realizar una definición adecuada al contexto actual de la enseñanza y de la docencia superior, para pasar luego a su tipología y, finalmente, al rol del docente; de esta manera se propondrá un contexto adecuado para definir las particularidades de esta actividad y sus necesidades específicas al momento de realizar el proceso de inducción.

El diccionario de la Real Academia Española (2011) define la palabra docente como "[persona] que enseña" o "perteneciente o relativo a la enseñanza" pero esta definición, sin duda, resulta excesivamente general y vaga frente a las características particulares del trabajo docente; de forma específica, del docente universitario. Para autores como Bozu (2010), el profesor universitario es la persona graduada que imparte la docencia en un universidad; pese a que esta definición es más particular al caso de estudio, todavía deja de comprender, por su generalidad, aspectos como la 


\section{María Belén Rueda Reyes \\ Propuesta teórica y metodológica del proceso de inducción laboral de personal docente en instituciones de educación superior}

profesionalización o la variedad de los tipos de institutos de educación superior. Finalmente, Martínez (1997) señala que el docente universitario es, por una parte, una persona con dominio en la materia que imparte y, por otra, un experto en el proceso de enseñanza-aprendizaje, cuya labor se realiza en la institución educativa superior; una definición que abarca ya elementos más específicos y cercanos a la que se considera en el presente artículo.

Con base en lo anotado y para realizar una definición adecuada al presente artículo, se considerarán tres elementos que, a criterio de la autora, configuran la actividad del docente universitario del que se trata en este documento: 1) la formalidad de la enseñanza; 2) la profesionalidad del docente; y, 3) la labor en el ámbito universitario.

La formalidad de la docencia se refiere a que la actividad se realiza en un contexto de aprendizaje institucionalizado (Colom, 2005), guiado por un currículo aceptado por entidad regulatoria competente (en el caso ecuatoriano, el Consejo de Educación Superior) y cuyos títulos finales (tercero o cuarto nivel) se encuentran reconocidos por el Estado donde se realiza la actividad de enseñanza e, incluso, por otros estados. En consecuencia, para definir al docente universitario en el presente artículo, se considerará que la labor de enseñanza se da en el ámbito formal.

Por otro lado, la docencia superior, en este artículo, se define como una actividad que requiere una formación académica y profesional para su ejercicio; formación que garantice, por una parte, el conocimiento de los contenidos que imparte el profesor y, por otra, la adquisición de habilidades y conocimientos vinculados al desarrollo de su trabajo de enseñanza para garantizar su calidad (Passos Alencastro y D'Ávila, 2008). De esta manera, el docente universitario está definido por su calidad de profesional, pudiendo serlo en el ámbito de la materia que imparte, en lo relativo a la actividad docente, o en ambas esferas.

Finalmente, el tipo de docente se analiza en este artículo se refiere al contexto de la educación superior, en cuanto a la actividad de transmitir y generar conocimiento. Frente a la amplitud de áreas que caracterizan la formación inicial e intermedia, la universitaria se diferencia por enfocarse en áreas que aseguren la preparación de profesionales en aspectos definidos pero que, en conjunto, abarcan la totalidad del conocimiento humano (Giustiniani y Carbajal, 2008). Es decir, la universidad (nótese la similitud etimológica con las palabras "universo" y "universal") pretende abarcar la amplitud de las ciencias, y lo hace a través de la estructura de facultades y escuelas. Adicionalmente, la enseñanza universitaria no se limita a la transmisión de conocimientos sino a generar nuevos a través de la investigación.

Con los antecedentes descritos, en el presente artículo se define como profesor universitario al trabajador dedicado a la enseñanza formal, con un perfil profesional ${ }^{3}$ relacionado a la materia que imparte y a la actividad docente, que realiza sus actividades laborales en una institución de educación superior, sea esta una universidad, una escuela politécnica, un instituto tecnológico, etcétera.

Ahora bien, la tipología de los docentes es diversa entre países, dependiendo de sus normas particulares e, incluso, varía entre los diferentes tipos de instituciones educativas superiores. En Ecuador, el Reglamento de Carrera y Escalafón del Profesor e Investigador del Sistema de Educación Superior establece dos tipos principales de trabajadores en el área académica de las universidades y escuelas politécnicas, institutos superiores técnicos, tecnológicos y pedagógicos, y conservatorios superiores de música y artes: personal académico, que incluye a los profesores e investigadores titulares y no titulares; y, personal técnico docente, que

\footnotetext{
${ }^{3}$ El perfil profesional se refiere al nivel académico que ostenta el docente y, en el caso ecuatoriano, determina incluso su capacidad para optar para diferentes categorías o tipos de trabajo.
} 


\section{María Belén Rueda Reyes \\ Propuesta teórica y metodológica del proceso de inducción laboral de personal docente en instituciones de educación superior}

está conformado por servidores de las instituciones de educación superior que cuenten con título profesional, experiencia y experticia en actividades de aprendizaje práctico. Con base en el ya mencionado Reglamento ecuatoriano, quienes realizan labor de docencia universitaria en Ecuador pueden ser titulares principales, agregados o auxiliares; mientras que los no titulares pueden ser honorarios, invitados u ocasionales (Consejo de Educación Superior, 2013). Los tipos de docentes de instituciones superiores en otros países adoptan denominaciones diversas como profesores de cátedra titulares, adjuntos 0 asociados, y docentes prácticos (Borgobello, Peralta y Roselli, 2010). En todo caso, se percibe que la labor docente puede ser interpretada como la impartición de cátedra y el desarrollo de habilidades y conocimientos prácticos entre los estudiantes, a lo que cabe añadir otras labores asociadas al docente como son la investigación o la vinculación con la sociedad (Ley Orgánica de Educación Superior, 2010).

\section{Particularidades a ser consideradas para la inducción a docentes universitarios}

Además de las diferencias que podrían adjudicarse a la diversa tipología del docente universitario, es necesario establecer sus múltiples roles, lo que permitirá determinar las particularidades de esta actividad. La práctica docente se manifiesta en varias dimensiones a través de las cuales se desarrolla su trabajo, entre las que la autora considera como más relevantes: a nivel personal, institucional, interpersonal y social. Para este análisis, se dejará de lado otras dimensiones en las que se desenvuelve la labor docente como la de didáctica y la de los valores (Contreras, 2003), no por considerarlas nimias sino porque dificultarían, aún más, el escenario de platear un modelo base para el desarrollo de las actividades de inducción a los trabajadores docentes universitarios.

En primer lugar, en una dimensión personal, se debe entender que el profesor es un ser humano con cualidades, características y personalidad; con motivaciones y conflictos internos, con proyectos y limitaciones (Collazo, Martínez y Liss, 2009). Para el desarrollo de actividades de inducción, es importante tener suficiente sensibilidad para considerar la propia historia personal, la experiencia profesional, la proyección profesional del nuevo docente, entre otras particularidades.

Por otra parte, en una dimensión institucional, la universidad constituye una organización heterogénea ${ }^{4}$ donde se despliegan las prácticas docentes. Se trata del escenario de socialización profesional, pues es allí donde los nuevos participantes del personal docente aprenden las normas y cultura (Mas Torelló, 2012). En este sentido, quien diseñe y realice el proceso de inducción debe considerar el enfoque estratégico, la organización y las condiciones específicas de la universidad.

Otra dimensión a considerar es la interpersonal. La práctica docente se fundamenta en las relaciones que se tejen entre los actores que intervienen en la vida universitaria: alumnos, docentes, personal administrativo y directores. Estas relaciones son complejas, pues se basan en las individualidades de cada miembro (Collazo, Martínez y Liss, 2009). La forma en que se desenvuelven estas relaciones constituye el clima institucional y delimita los espacios de participación interna y la comunicación. Según lo descrito, el proceso de inducción debería considerar el ambiente institucional para ser desarrollado de manera óptima.

Finalmente, la dimensión social se refiere a que la labor del maestro, por su función de formador, confirma y mantiene las estructuras sociales, pero también funciona como vía para su transformación y desarrollo (Ibarra, 2008). El rol del docente es tan complejo como la propia estructura social en la que se desenvuelve,

\footnotetext{
${ }^{4}$ Heterogénea en cuanto se diferencia de otras instituciones de educación superior, y es diversa en su conformación de facultades y escuelas. 


\section{María Belén Rueda Reyes \\ Propuesta teórica y metodológica del proceso de inducción laboral de personal docente en instituciones de educación superior}

con las variables culturas particulares. El análisis de la dimensión social del trabajo del docente universitario incluye una reflexión sobre las expectativas de la comunidad, las diferencias de género, las desigualdades en el ingreso, el contexto geográfico, entre otros. El proceso de inducción debe considerar esta dimensión y sus particularidades en el momento de su diseño y desarrollo.

Las particularidades anotadas en cada una de las dimensiones en las que se desenvuelve el docente universitario configuran un escenario complejo para generalizar un modelo metodológico para el proceso de inducción a nuevos profesores de educación superior. Sin embargo, se considera que existe la posibilidad de plantear lineamientos generales a ser tomados en cuenta y que se presentan en la siguiente sección.

\section{Propuesta de proceso de inducción a docentes universitarios}

Para el desarrollo de la propuesta, se han considerado dos dimensiones que justifican, metodológicamente, tanto su desarrollo como su eventual adaptación y aplicación en instituciones de educación superior: la dimensión de forma y la dimensión de fondo.

En lo referente a la forma, se ha considerado ofrecer dentro de la propuesta cuatro modelos documentales: flujograma del proceso, guía de inducción general, guía de inducción específica y hoja de evaluación del proceso a ser aplicada a los docentes de nuevo ingreso ${ }^{5}$. El mantenimiento de documentación en las instituciones es indispensable para que sirva como insumo para el desarrollo de acciones de control y evaluación, dentro de modelos como los de mejora continua (Nava y Jiménez, 2005).

El modelo propuesto de flujograma cuenta con elementos formales mínimos como son: un espacio para el nombre y el logotipo de la institución, nombre del proceso, codificación del proceso o subproceso, número de versión, fecha de entrada en vigencia y nombres de los responsables de su elaboración y aprobación (Kendall, 2005). Por otra parte, los modelos de hojas de inducción, tanto general como específica, y de evaluación cuentan con espacios de identificación y de instrucciones, que facilitan la aplicación de las herramientas, archivo y uso posterior (Russo, 2009).

En lo referente al fondo de la propuesta, las acciones del proceso de inducción suelen contemplar, en términos generales, la información al nuevo ingreso sobre la situación del empleo, las políticas y reglas de la compañía, la remuneración, la cultura corporativa, el trabajo en equipo, el desarrollo del empleado y la socialización (Mondy, 2010). Aunque no se puede establecer un formato fijo del proceso, para el propósito del presente artículo se recomienda iniciar con acciones de bienvenida de tal manera que el nuevo colaborador elimine la ansiedad propia de la incorporación al cargo. Posteriormente, se deberá realizar una explicación general de la organización (historia, enfoque estratégico, organigrama, etc.), para pasar luego a información específica de las funciones, responsabilidades y relaciones del puesto de trabajo. Finalmente, se realiza la presentación oficial ante el superior jerárquico, los compañeros del mismo nivel y los subordinados (en caso de haberlos), así como la entrega de bienes muebles, llaves, tarjetas, equipos de protección y otros que sean de uso cotidiano al puesto de trabajo (Bozu, 2010).

Con base en lo descrito, se presenta a continuación una propuesta metodológica para el proceso de inducción a docentes universitarios, para lo cual se establecen las siguientes consideraciones generales:

\footnotetext{
${ }^{5}$ Se han considerado estos elementos documentales con base en aportes de autores como Ramírez (2004), Chiavenato (2009) y Grados (2013). Las instituciones educativas pueden incluir otros documentos para el proceso o prescindir de alguno de los propuestos, con base en sus requerimientos particulares 


\section{María Belén Rueda Reyes \\ Propuesta teórica y metodológica del proceso de inducción laboral de personal docente en instituciones de educación superior}

- En la esfera estratégica, se plantea que el departamento de talento humano (recursos humanos u otra denominación que adopte) de la institución superior establezca los objetivos, las estrategias y las tácticas del proceso de inducción al personal docente, conforme a sus competencias (Chiavenato, 2007). Este conjunto de objetivos deben considerar, en su diseño, el enfoque estratégico general de la institución de manera expresa y contar con aprobación previa a la socialización entre las autoridades de las diferentes facultades ${ }^{6}$.

- En la esfera administrativa, se propone que el proceso de inducción sea planificado, organizado y controlado desde el área de recursos humanos. El control, por medio de evaluaciones que ofrezcan medios de verificación, servirá para un proceso de mejora continua (Nava y Jiménez, 2005). Para las funciones descritas, el departamento deberá diseñar documentos adecuados de guía, seguimiento y evaluación, para lo que se pueden usar como modelos los que se presentan en el presente artículo.

- A nivel operativo, la ejecución debería realizarse en conjunto entre el área de gestión del talento humano y un representante designado por la facultad a la que ingresa el nuevo docente, en dos fases. En la primera etapa, la unidad de talento humano de la institución educativa superior debería conducir la inducción para brindar información en el ámbito general (condiciones del empleo, enfoque estratégico de la universidad, normas y reglamentos de la institución, compensaciones, estructura orgánica, etc.); mientras que, en la segunda fase, el representante de la facultad realizaría la inducción en el ámbito específico (presentación de las instalaciones, introducir al equipo de trabajo, información específica sobre las funciones, entre otros elementos) (Mondy, 2010). Estas actividades pueden facilitarse con la entrega de folletos informativos a los nuevos ingresos que incluyan misión, visión y objetivos institucionales, información histórica, organigramas, reglamentos internos, etc.

- El conjunto y orden de las acciones del proceso de inducción propuesto ha sido adaptado por la autora con base en aportes de Bozu (El profesorado universitario novel: estudio teórico de su proceso de inducción o socialización profesional, 2010), ITT (Procedimiento de inducción al personal de nuevo ingreso, 2018) y Grados (Reclutamiento, selección, contratación e inducción del personal, 2013); todos, documentos que han servido previamente para argumentar el desarrollo del presente artículo. Así mismo, se ha tomado en cuenta como eje central para el diseño del flujo del proceso la relación entre la inducción y otros procesos del área de talento humano propuesto por Chiavenato (2009) y que fue ilustrado en la Figura 1. De manera adicional, la autora ha considerado su experiencia previa en el estudio de relación entre la inducción y el desempeño docente (Rueda, 2015).

- Se debe considerar una aproximación teórica del proceso de inducción, sea la presentada en este artículo u otra que se fundamente en la extensa literatura relacionada con el tema. En ese sentido, tanto el diseño como las modificaciones futuras a la metodología de inducción que se aplique en las universidades deben ser elaboradas por un profesional en el área.

Con las consideraciones generales anotadas, a continuación, en la Figura 3 , se presenta un modelo de diagrama de flujo de un posible proceso de inducción a docentes universitarios:

\footnotetext{
${ }^{6}$ Sin duda, se deberá atender a la estructura orgánica propia de la universidad donde se aplicará la propuesta. Será diferente la aplicación en una institución que cuente con un departamento único de recursos humanos frente a otra que posea, por su tamaño, unidades adicionales de talento humano en cada facultad.
} 
Propuesta teórica y metodológica del proceso de inducción laboral de personal docente en instituciones de educación superior

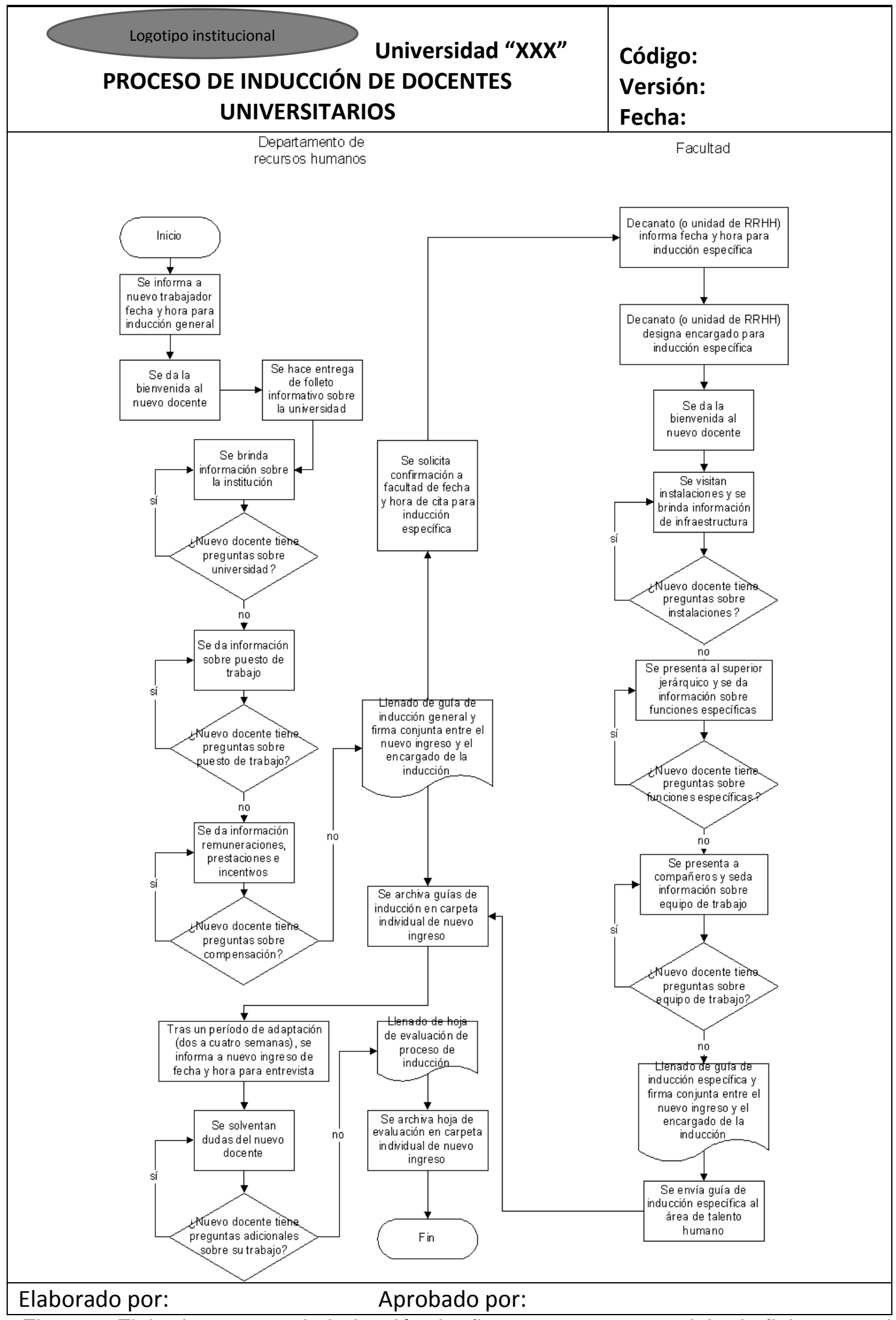

Figura 3. Flujo de proceso de inducción. La figura expone un modelo de flujograma de proceso de inducción a aplicarse a docentes universitarios y que puede adaptarse a las necesidades específicas de la institución. 


\section{María Belén Rueda Reyes \\ Propuesta teórica y metodológica del proceso de inducción laboral de personal docente en instituciones de educación superior}

Dentro del flujograma propuesto, se hace mención al llenado y suscripción de dos guías de inducción, que podrían adoptar una forma como la que se propone en las Figuras 4 y 5 a continuación:

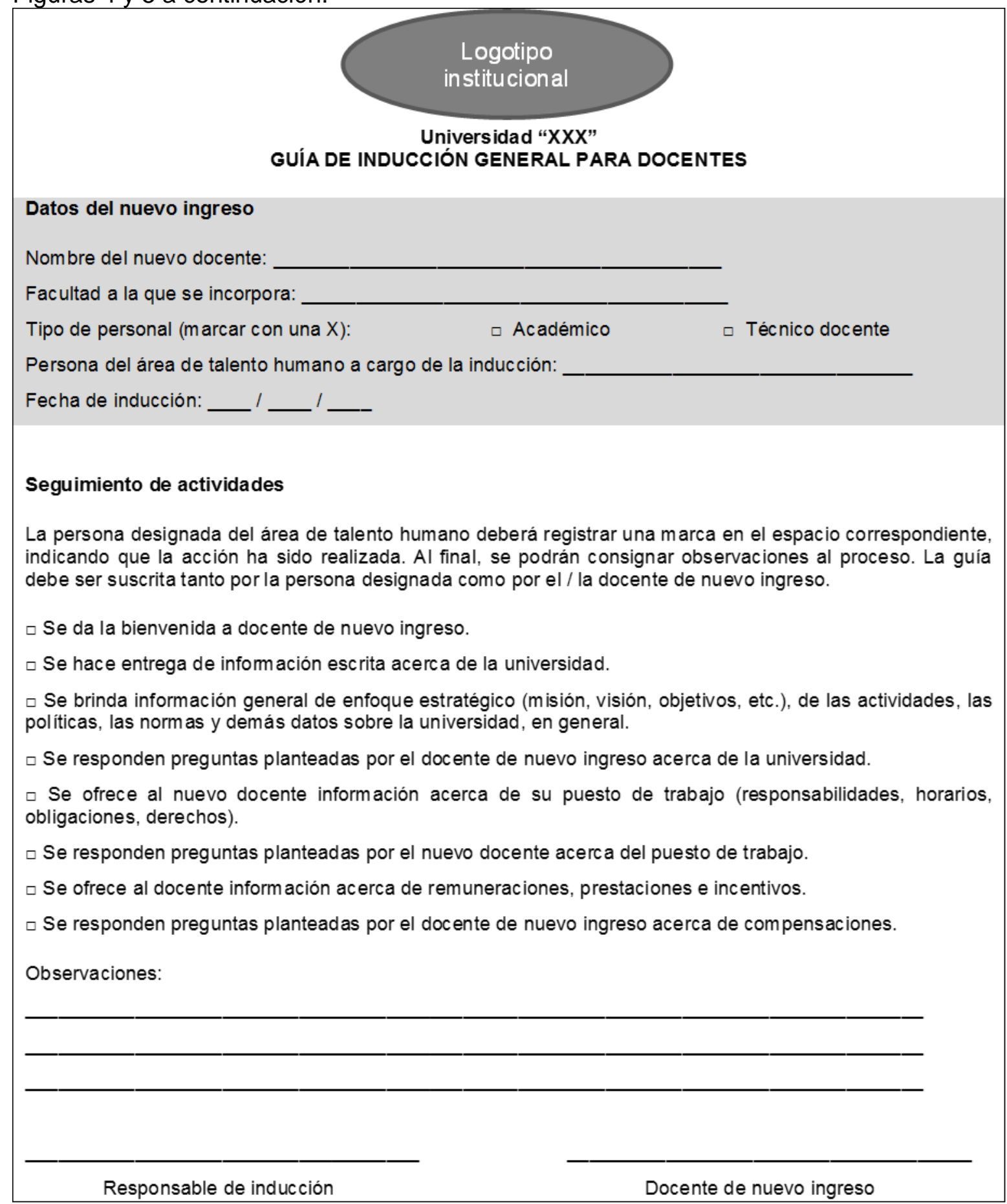

Figura 4. Guía de inducción general. La figura presenta un modelo de guía a ser aplicada por un designado del área de recursos humanos en la inducción a nuevos docentes universitarios que puede adaptarse a las necesidades específicas de la organización. 


\section{María Belén Rueda Reyes \\ Propuesta teórica y metodológica del proceso de inducción laboral de personal docente en instituciones de educación superior}

Figura 5. Modelo de guía de inducción específica

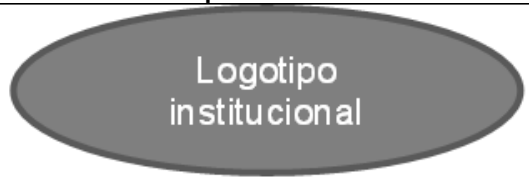

Universidad " $X X X$ "

GUÍA DE INDUCCIÓN ESPECÍFICA PARA DOCENTES

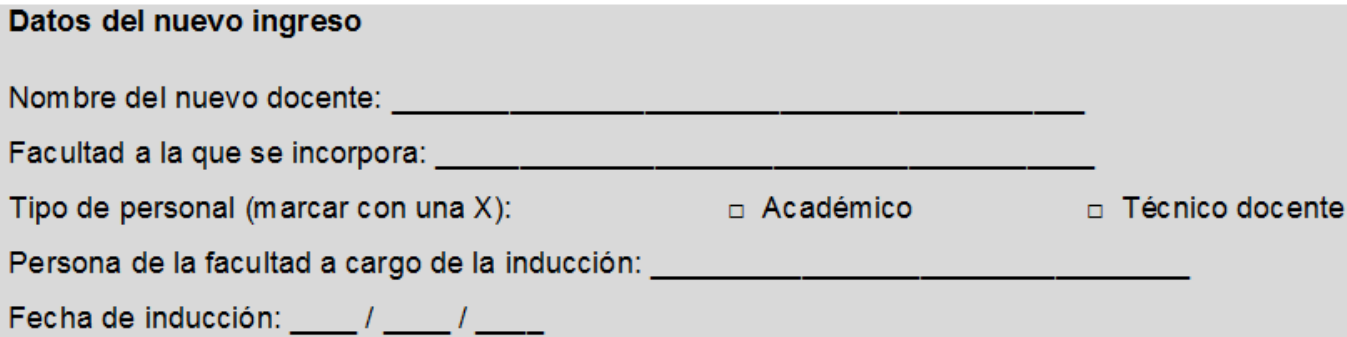

La persona designada de la facultad respectiva deberá registrar una marca en el espacio correspondiente, indicando que la acción ha sido realizada. Al final, se podrán consignar observaciones al proceso. La guía debe ser suscrita tanto por la persona designada como por el / la docente de nuevo ingreso y entregada al área de talento humano de la universidad.

$\square$ Se da la bienvenida a docente de nuevo ingreso.

$\square$ Se realiza una visita guiada a todas las instalaciones de la facultad, con especial interés en el área de aulas donde desempeñará su trabajo en nuevo docente.

$\square$ Se brinda información general sobre las instalaciones de la facultad y la universidad.

$\square$ Se responden preguntas del docente de nuevo ingreso acerca de la infraestructura de la universidad.

$\checkmark$ Se presenta al nuevo docente su (sus) superior (superiores) jerárquicos.

口 Se ofrece al nuevo docente información acerca de sus funciones específicas en el puesto de trabajo.

$\square$ Se responden preguntas planteadas por el nuevo docente acerca de sus funciones especificas.

$\square$ Se presenta al nuevo docente entre los compañeros y equipo de trabajo.

- Se ofrece al docente información acerca de las dinámicas de grupo, los equipos de trabajo y la relación entre compañeros.

$\square$ Se responden preguntas planteadas por el docente de nuevo ingreso acerca de los grupos de trabajo.

Observaciones:

Figura 5. Guía de inducción específica. La figura presenta un modelo de guía a ser aplicada por un designado del área de recursos humanos en la inducción a nuevos 


\section{María Belén Rueda Reyes \\ Propuesta teórica y metodológica del proceso de inducción laboral de personal docente en instituciones de educación superior}

docentes universitarios que puede adaptarse a las necesidades específicas de la organización.

Finalmente, se propone, a manera de ejemplo, la utilización de una hoja de evaluación al proceso de inducción a ser llenado por el nuevo ingreso y que podría adoptar el modelo presentado en la Figura 6:

\section{Logotipo \\ institucional \\ Universidad "XXX" \\ HOJA DE EVALUACIÓN DEL PROCESO DE INDUCCIÓN PARA DOCENTES}

El propósito de la presente evaluación es conocer sus criterios acerca del proceso de inducción que se aplicó al momento de su reciente ingreso a la institución. Sus comentarios honestos ayudarán a la universidad a mejorar en el futuro la forma en que se orienta a los docentes al inicio de su trabajo en la organización.

Datos del nuevo ingreso

Nombre del docente:

Facultad a la que se incorporó:

Tipo de personal (marcar con una X):

$\square$ Académico

- Técnico docente

Fecha de evaluación

Marque la respuesta con una $\mathrm{X}$.

Al iniciar su trabajo en la universidad, usted:

S

No

¿Recibió una bienvenida por parte de un representante de talento humano?

¿Recibió información general escrita acerca de la universidad?

¿Se le informó acerca de la historia, las políticas, las normas, etc., de la institución?

¿Recibió respuestas satisfactorias frente a sus preguntas respecto de la institución?

¿Se le informó acerca de las características del puesto de trabajo?

¿Recibió respuestas satisfactorias a sus preguntas respecto del puesto de trabajo?

¿Se le dio información acerca de las remuneraciones, incentivos y otras compensaciones?

¿Obtuvo respuestas adecuadas frente a sus preguntas sobre las compensaciones?

¿Recibió una bienvenida por parte de un representante de la facultad a la que usted ingresó?

¿Fue acompañado en una visita a las instalaciones de la facultad?

¿Recibió información acerca de los edificios e infraestructura de la facultad?

¿Obtuvo respuestas satisfactorias frente a sus preguntas sobre las instalaciones?

¿Fue presentado a su superior jerárquico inmediato?

¿Recibió información acerca de la organización y las líneas jerárquicas?

¿Recibió respuestas adecuadas frente a sus preguntas respecto de las relaciones jerárquicas?

¿Fue presentado ante miembros de la institución, compañeros y participantes del equipo?

¿Recibió información acerca de las relaciones entre los equipos de trabajo?

¿Sus preguntas sobre los equipos de trabajo y relaciones entre personas fueron respondidas?

Sugerencias que quiera compartir acerca del proceso de inducción:

\section{Docente}

Figura 6. Hoja de evaluación del proceso de inducción. La figura presenta un modelo de guía a ser aplicada por un designado del área de recursos humanos en la inducción 


\section{María Belén Rueda Reyes \\ Propuesta teórica y metodológica del proceso de inducción laboral de personal docente en instituciones de educación superior}

a nuevos docentes universitarios que puede adaptarse a las necesidades específicas de la organización.

\section{CONCLUSIONES}

Como principal conclusión del artículo se puede anotar que la inducción es un proceso de la gestión del talento humano que favorece la adaptación de los nuevos trabajadores a la cultura y las actividades de la empresa. El proceso, cuando es adecuado, presenta beneficios tanto a las organizaciones (a través de una menor rotación de personal, reducción de costos de capacitación, mejoramiento de la competitividad) como a los trabajadores (mejor ambiente laboral, desarrollo de las capacidades, entre otras).

Los beneficios de un apropiado proceso de inducción no son extraños a trabajos de alta complejidad y heterogeneidad como la de los docentes universitarios; heterogeneidad que radica no solo en los tipos diversos de docentes (según las normas específicas de cada institución educativa o las impuestas por la legislación vigente), sino por las dimensiones personales, institucionales, interpersonales y sociales de cada profesor de nuevo ingreso.

El desarrollo del presente artículo respondió, parcialmente, a los resultados obtenidos del estudio "Incidencia del proceso de inducción en el desempeño docente de la Facultad de Arquitectura, Artes y Diseño de la Universidad Tecnológica Equinoccial" (Rueda, 2015). Pese a los resultados de dicho estudio, el presente artículo permitió concluir que un adecuado trabajo de inducción resulta beneficioso para los docentes, la institución educativa y para toda la comunidad universitaria, con base en aportes de autores como Bozu (2010), Perea (2006), Colmenares (2008), Chiavenato (2009), Mondy (2010), entre otros. En vista de lo señalado, se puede concluir que los resultados de Rueda (2015) respondieron a una limitada aproximación metodológica (en la que se trató de establecer una relación con una sola variable de análisis) sin considerar otras ligadas a la gestión de talento humano además del hecho de que la evaluación del proceso de evaluación en el estudio de 2015 se basó en encuestas aplicadas a docentes y no en documentos (como los que se proponen en este este artículo), lo que pudo tener un efecto negativo en la validez de la investigación.

Pese a las dificultades que presenta desarrollar un procedimiento normalizado de inducción en las instituciones educativas superiores, los departamentos de talento humano deben implementar acciones (planeación, diseño metodológico, organización, ejecución compartida con las facultades a las que ingresa el nuevo docente, evaluación y mejoramiento), con el fin de beneficiar a los docentes universitarios, a las instituciones; y, a través de ellos, a la sociedad en general. Varias normas internacionales (como la familia de normas ISO) recomiendan la documentación de los procesos, por lo que el presente documento propone modelos de caracterización y flujograma de proceso de inducción, así como guías general y específica de inducción, y de una hoja de evaluación del proceso. Tanto las acciones como la documentación del proceso deberán responder a las características particulares observadas de los profesores, de su relación con la institución, el ambiente de trabajo e, incluso, la sociedad. 


\section{BIBLIOGRAFÍA}

Ley Orgánica de Educación Superior, Registro Oficial No. 298 (Función Ejecutiva 12 de octubre de 2010).

Alles, M. (2006). Dirección Estratégica de Recursos Humanos. Buenos Aires: Granica.

Borgobello, A., Peralta, N. y Roselli, N. (2010). El estilo docente universitario en relación al tipo de clase y a la disciplina enseñada. Liber. v.16 n.1 Lima ene./jun., 7-16.

Bozu, Z. (2010). El profesorado universitario novel: estudio teórico de su proceso de inducción o socialización profesional. Revista Electrónica de Investigación y Docencia Reid. ISSN: 1989-2446, 55-72.

Chiavenato, I. (2007). Administración de Recursos Humanos. México: Mc Graw Hill.

Chiavenato, I. (2009). Gestión del Talento Humano. México D.F.: Mc Graw Hill.

Collazo, M., Martínez, D., y Liss, M. (2009). Dimensiones del trabajo docente. Educ. Soc., Campinas, Vol 30. N. 107, 389-408.

Colom, A. (2005). Continuidad y complementariedad entre la educación formal y no formal. Revista de Educación. No. 338. 9-22.

Consejo de Educación Superior. (16 de julio de 2013). Reglamento de Carrera y Escalafón del Profesor e Investigador del Sistema de Educación Superior. Obtenido de Reglamento 037-265: http://www.ces.gob.ec/doc/gaceta_ces/reglamento/reglament\%20037-265.pdf

Contreras, J. (2003). La práctica docente y sus dimensiones. VALORAS UC, 1-3.

Giustiniani, R. y Carbajal, L. (2008). Universidad, democracia y reforma: algunas reflexiones y una propuesta. Buenos Aires: Prometeo Libros.

Grados, J. (2013). Reclutamiento, selección, contratación e inducción del personal:. México D.F.: El Manual Moderno.

Ibarra, A. (2008). La función del docente: entre los compromisos éticos. Bogotá: Universidad Pedagógica Nacional Colombia.

ITT. (2018). Procedimiento de inducción al personal de nuevo ingreso. Obtenido de Instituto Tecnológico de Tlajomulco: http://www.ittlajomulco.edu.mx/2018/sgc/proceso_estrategico_admon_recursos /ITTJ-AD-PO-004-INDUCCION-PERSONAL-NUEVO-INGRESO//ITTJ-AD-PO004\%20PROC\%20INDUCCION\%20AL\%20PERSONAL\%20DE\%20N.I..pdf

Kendall, K. (2005). Análisis y diseño de sistemas. Obtenido de Books: https://books.google.com.ec/books?id=5rZA0FggusCypg=PT312ydq=formalidades+de+diagrama+de+flujoyhl=es419ysa $=$ Xyved=0ahUKEwiwgKPipMnaAhVMq1MKHavbAoMQ6AEIODAD\#v=0

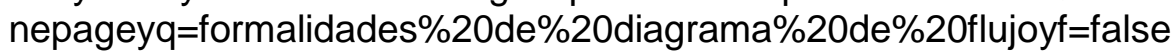


Propuesta teórica y metodológica del proceso de inducción laboral de personal docente en instituciones de educación superior

Marrou, H.-I. (2004). Historia de la educación en la antigüedad. Madrid: Akal.

Mas Torelló, Ó. (2012). Las competencias del docente universitario: la percepción del alumno, de los expertos y del propio protagonista. Revista de Docencia Universitaria. Vol. 10. No. 2, 299-318.

Mejía, A., Jaramillo, M. y Bravo, M. (2006). Formación del talento humano: factor estratégico para el desarrollo de la productividad y la competitividad sostenibles en las organizaciones. Obtenido de Revista científica Gillermo de Ockham. Vol 4. No. 1: http://dx.doi.org/10.21500/22563202.487

Mondy, W. (2010). Administración de recursos humanos. México: Pearson.

Nava, V. M. y Jiménez, A. R. (2005). ISO 9000:2000: estrategias para implantar la norma de calidad para la mejora. México: Limusa.

Passos Alencastro, I. y D'Ávila, C. (2008). Profesión Docente. San Pablo, Brasil: Papirus Editora.

Pérez, J. (2010). Gestión por procesos. Madrid: ESIC.

Puchol, L. (2007). Dirección y Gestión de Recursos Humanos. Madrid: Díaz de Santos.

RAE. (2011). Diccionario de la Real Academia Española. Madrid: Espasa.

Rodríguez, D. (2006). Gestión organizacional: elementos para su estudio. México D.F.: Alfaomega.

Rueda, M. (2015). Incidencia del proceso de inducción en el desempeño docente de la Facultad de Arquitectura, Artes y Diseño de la Universidad Tecnológica Equinoccial. Quito: Universidad Tecnológica Equinoccial.

Russo, P. (2009). Gestión documental en las organizaciones. Barcelona, España: El Ciervo. 
Propuesta teórica y metodológica del proceso de inducción laboral de personal docente en instituciones de educación superior

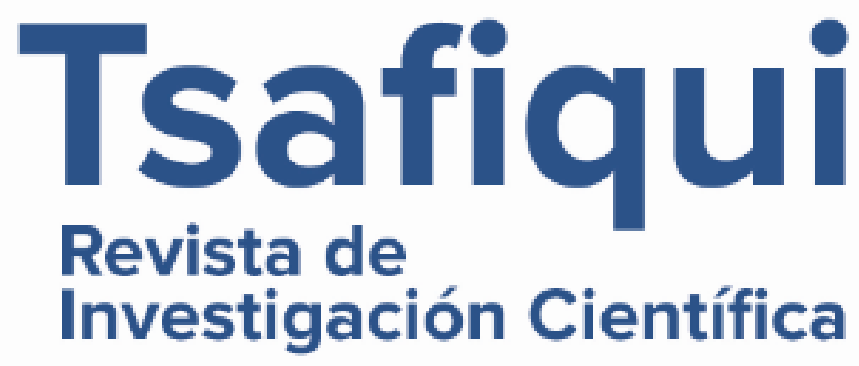

